\title{
Improving Access to Rheumatologists: Use and Benefits of an Electronic Consultation Service
}

\author{
Krista Rostom, C. Douglas Smith, Clare Liddy, Amir Afkham, and Erin Keely
}

ABSTRACT. Objective. To describe the use and benefits of an innovative eConsult service to improve access to rheumatologists.

Methods. There were 225 eConsults directed to rheumatology that were categorized by type of question and effect on face-to-face referral rates.

Results. The median response time by the rheumatologists was 1.9 days. Clinical questions included drug treatment $(34 \%)$, diagnosis $(26 \%)$, or management $(14 \%)$. Osteoporosis was the most common diagnosis $(22 \%)$, followed by pain in multiple joints $(11 \%)$, and polyarthritis (10\%). A face-to-face referral was avoided in $38 \%$ of cases.

Conclusion. There are clinical questions that can be answered quickly by an eConsult, improving access to rheumatologists. (First Release November 1 2017; J Rheumatol 2018;45:137-40; doi:10.3899/jrheum.161529)

Key Indexing Terms: RHEUMATOLOGY

\section{REMOTE CONSULTATION}

\section{TELEMEDICINE}

The rising prevalence of rheumatic diseases and the static number of rheumatologists are major concerns for access to care $^{1,2,3}$. In Canada, wait times for specialist consults for arthritis or rheumatism were longer than for all other conditions, with $31 \%$ taking over 3 months, and the wait is a major barrier to providing adequate care ${ }^{4,5}$. Improving access is a priority for professional groups, including the Canadian Rheumatology Association 3,5,6,7.

Virtual consultations, including technology-enhanced patient-provider visits (telemedicine), and electronic consults (eConsults) may replace a face-to-face visit or enhance the effectiveness of a future visit. Unlike telemedicine, an eConsult does not require the patient to be present and the providers communicate asynchronously, eliminating the need to align schedules. Aside from telemedicine, there are few studies looking at virtual consultation in rheumatology. One

From the Department of Medicine, University of Ottawa; Division of Rheumatology, The Ottawa Hospital; Department of Family Medicine, University of Ottawa; C.T. Lamont Primary Health Care Research Centre, Bruyère Research Institute; Champlain Local Health Integration Network; Ottawa Hospital Research Institute, Clinic; Division of

Endocrinology/Metabolism, Ottawa Hospital, Ottawa, Ontario, Canada. Supported by the University of Ottawa Department of Medicine, Bruyère Research Institute, and the Royal College of Physicians and Surgeons of Canada.

K. Rostom, MD, Department of Medicine, University of Ottawa; C.D. Smith, MD, FRCPC, Department of Medicine, University of Ottawa, and Division of Rheumatology, The Ottawa Hospital; C. Liddy, MD, MSc, CCFP, FCFPC, Department of Family Medicine, University of Ottawa, and C.T. Lamont Primary Health Care Research Centre, Bruyère Research Institute; A. Afkham, Champlain Local Health Integration Network;

E. Keely, MD, FRCPC, Department of Medicine, University of Ottawa, Ottawa Hospital Research Institute, Clinic, and Division of Endocrinology/Metabolism, Ottawa Hospital.

Address correspondence to Dr. E. Keely, 1967 Riverside Dr., Rm 4-01, Ottawa, Ontario K1H 7W9, Canada.E-mail: ekeely@toh.on.ca

Accepted for publication August 16, 2017.
Canadian study compared conventional, e-mail, and videoconferencing consults in 3 rural communities. Primary care providers (PCP) preferred videoconferencing; however, e-mail also received positive responses ${ }^{8}$. A single site American study demonstrated that $25 \%$ of eReferrals could be addressed without a clinic visit when the rheumatologist and PCP communicated directly through an electronic system $^{9}$.

The purpose of this paper is to describe the use, benefits, and effect on the need for face-to-face consultations of rheumatology eConsults completed through the Champlain Building Access to Specialists through eConsultation (BASE) service.

\section{MATERIALS AND METHODS}

Champlain BASE eConsultation Service. The Champlain BASE eConsult system is an asynchronous, secure Web-based application whereby a PCP may submit patient-specific clinical questions to multiple specialty services ${ }^{10,11}$. The case is assigned to an individual specialist who is expected to respond within 1 week. Laboratory results or photos can be attached, and specialists may request more information, provide a recommendation, or suggest a face-to-face referral. There may be iterative communication between the specialist and PCP. The PCP completes a mandatory closeout survey. One question asks whether the eConsult confirmed their originally chosen course of action, or suggested a new or additional course of action. Another asks the PCP to identify whether they had originally contemplated a referral, and whether they needed to refer the patient after receiving the eConsult response. The specialist self-reports the time required to answer the eConsult and is paid a prorated hourly rate. Since the launch in April 2011, over 1200 PCP have enrolled. There are 102 specialty groups available and over 24,000 eConsults were completed. User satisfaction is high, with PCP commonly citing promptness, quality of replies, and added education from specialist responses as benefits ${ }^{10,12}$.

Setting and participants. The Champlain region of Eastern Ontario has a population of 1.2 million $^{10}$. Rural PCP provided $12 \%$ of eConsults.

Data collection. Data were prospectively collected, stored securely, and then retrospectively accessed for analysis. This included specialist response time,

Personal non-commercial use only. The Journal of Rheumatology Copyright $\odot$ 2018. All rights reserved. 
time taken to complete the case, and the PCP closeout survey. A log containing the full exchange was saved.

Ethics. Ethics approval was received from the Ottawa Hospital Research Ethics Board (2009848-OH1).

Question categorization. All eConsults were reviewed and categorized retrospectively by clinical content and type of question by a single reviewer. A predefined list of 31 clinical diagnoses (reached by consensus) and types of clinical questions (based on validated question taxonomy) was created ${ }^{13}$ Diagnoses that represented under $3 \%$ of the cases were combined into "other".

\section{RESULTS}

Of the 5597 eConsults completed from April 15, 2011, to January 31, 2015, there were 225 (4\%) directed to rheumatology. One rheumatologist answered $71 \%$ of cases, while 2 other rheumatologists answered $21 \%$ and $8 \%$, respectively. Cases were submitted by 125 different clinicians, 17 (8\%) of whom were nurse practitioners. The rheumatologist requested further information before answering in 12 cases (5\%). Average response time was 2.8 days (median 1.9 days). Self-reported time to complete the eConsult was under 10 $\min$ in $50 \%, 10-15 \min$ in $39 \%, 15-20 \mathrm{~min}$ in $10 \%$, and over $20 \mathrm{~min}$ in $<1 \%$ of cases.

The eConsult changed the clinical path for many patients. New recommendations for the course of action occurred in $54 \%$ of cases, and in $38 \%$, a referral was avoided (Table 1). There was a diverse set of diagnoses and symptoms, with osteoporosis $(22 \%)$, pain in multiple joints $(11 \%)$, and polyarthritis (10\%) being the most common (Table 2). Osteoporosis, osteoarthritis (OA), and crystal arthritis eConsults had high rates of referral avoidance, while osteoporosis and abnormal serologic marker without joint symptoms both had high rates of referral that was not originally contemplated and still not needed.

The most common question types were drug treatment $(34 \%)$, diagnosis $(26 \%)$, management $(14 \%)$, or there was more than 1 question and they were thus unclassifiable (17\%, Table 3). Drug treatment questions were most commonly about drug of choice (12\%) and "indications or goals of treating a particular condition" $(11 \%)$.

\section{DISCUSSION}

Our study demonstrated that the eConsult was highly regarded by PCP and can improve access to rheumatology advice in a timely manner, with most eConsults being answered in under 3 days. In addition, 38\% of these patients are no longer on our waitlist because a traditional referral was avoided. The benefits of eConsult are especially needed in rheumatology, where long wait times and manpower issues are prevalent.

While not the same as an eConsult, preconsultation exchange uses a similar strategy with information exchange between PCP and specialist, to facilitate triaging of the consultation, to redirect if more appropriate, or to provide suggestions or further investigation. Within rheumatology, 2 groups have looked at preconsultation exchange. In 1 group, a rheumatologist reviewed either faxed or electronic medical records before appointment scheduling and found that $41 \%$ of referred patients did not require a rheumatology consultation ${ }^{14}$. A second group identified that $37 \%$ of the eReferrals that underwent electronic preexchange were resolved without face-to-face consultation 9 .

Our rate of $38 \%$ of eConsults being resolved without traditional face-to-face consultation is remarkably similar to the rates of resolved consultations described by these groups. Independent groups of rheumatologists believe they can safely provide consultation without a face-to-face appointment and the similar referral avoidance rates suggest there is a real potential for eConsult services to shorten waitlists. We are unable to determine whether patient outcomes are com-

Table 1. Outcome of eConsults based on the PCP close-out survey.

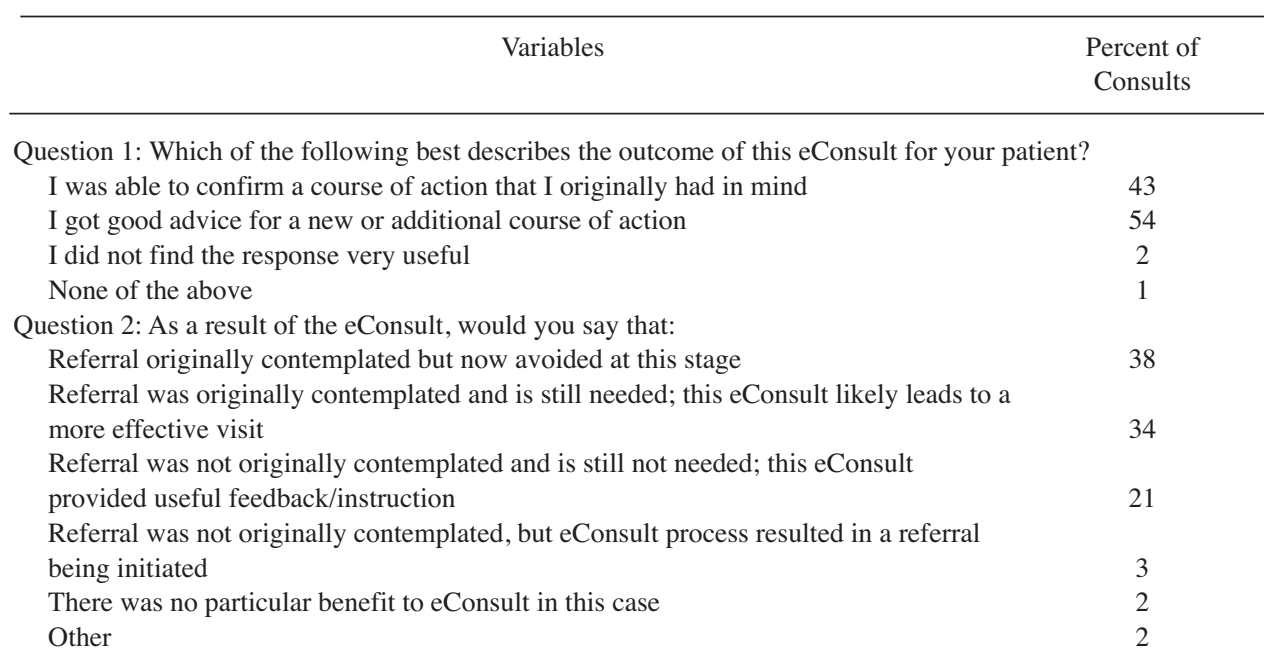

PCP: primary care physician. 
Table 2. eConsults categorized by diagnosis or symptom, then subcategorized by referral outcome. Values are n (\%).

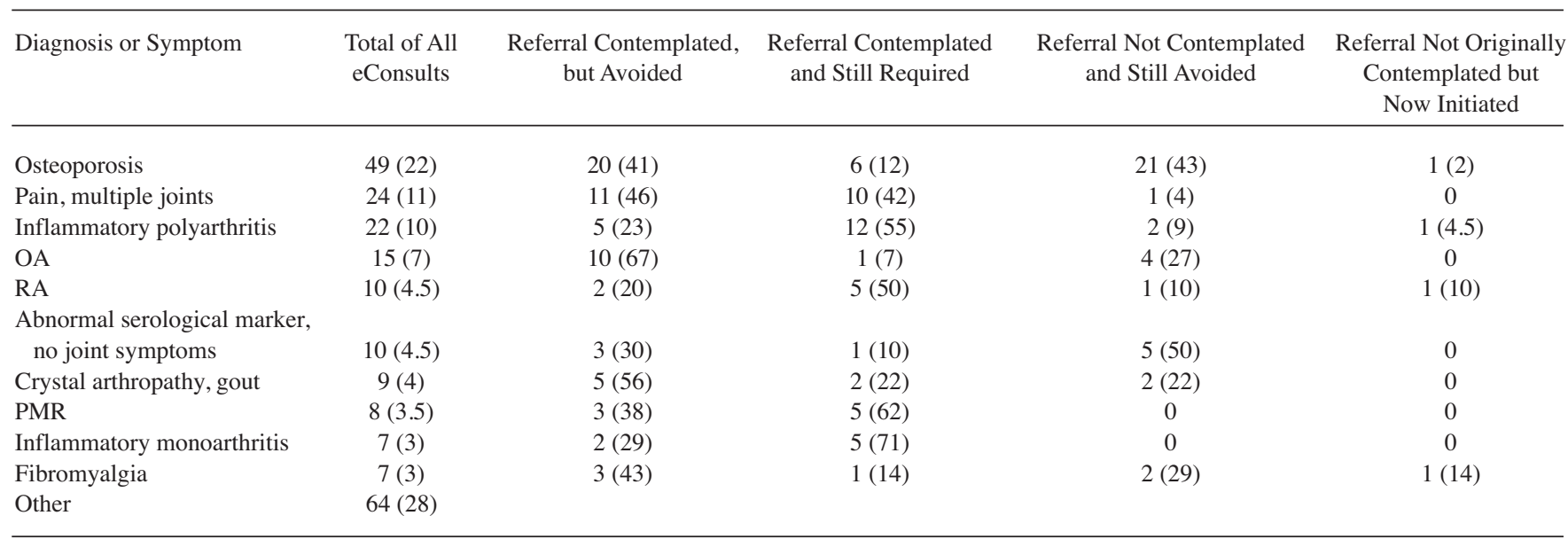

OA: osteoarthritis; RA: rheumatoid arthritis; PMR: polymyalgia rheumatica.

Table 3. Classification of clinical questions of submitted eConsults.

\begin{tabular}{lc}
\hline Clinical Question & Percent of Consults \\
\hline Diagnosis & \\
Interpretation of a laboratory test & 9.8 \\
Interpretation of an image report & 2.2 \\
Interpretation of a clinical finding & 4.4 \\
Other & 3.1 \\
What test to choose & 6.2 \\
Drug treatment & \\
Other & 1.8 \\
Drug of choice & 12.4 \\
Adverse effects of drugs & 3.6 \\
How to prescribe a particular drug & 3.1 \\
Indications/goals of treating a particular condition & 11.1 \\
Interactions between drugs & 1.8 \\
Management & \\
Other providers available & 0.4 \\
General management question & 5.3 \\
Should I refer & 8.4 \\
More than 1 question (unclassifiable) & 17.3 \\
No specific question & 6.7 \\
Procedure & \\
Indications & 1.3 \\
Other & 0.9 \\
\hline
\end{tabular}

parable to those patients referred for a face-to-face visit, and further studies are required to address this.

Our characterization of the clinical questions of the eConsults provides insight into the service use. With higher rates of referral avoidance in osteoporosis, OA, and crystal arthritis, it may be beneficial to highlight the benefits with these diagnoses when implementing an eConsult service. We do not have comparable data on the scope of diagnosis of the traditional faxed consultations, but this is an area of interest and future study.

Osteoporosis and abnormal serological marker without joint symptoms cases have a high rate of referral not origi- nally contemplated and still avoided, suggesting PCP are seeking further guidance on these diagnoses without contemplating a traditional referral. These may be areas to target for PCP continuing medical education.

There were a few eConsults pertaining to inflammatory arthritis, which were categorized as referral avoided. Because care from a rheumatologist is the standard of care in chronic inflammatory arthritis, we reviewed these eConsults for quality assurance. Three cases pertained to patients with rheumatoid arthritis who were taking immunosuppressants, suggesting a rheumatologist was already involved; however, the PCP question related to medication side effects or interactions (e.g., whether shingles vaccine was contraindicated for a patient taking hydroxychloroquine). Two cases of polyarthritis were generally self-limited diseases (Parvovirus B19 and Lofgren syndrome), while the presence of inflammation was unclear in other cases, with the rheumatologist suggesting an investigation and referral if needed. Two cases of monoarthritis were classified as avoided, with 1 sternoclavicular monoarthritis directed to interventional radiology, and the other a question about distinguishing arthritis from ligamentous injury.

Our study has limitations, however. The geographical limitations of a single health region may make the results not generalizable. As eConsult services continue to expand, it is hoped that others will validate our findings in other jurisdictions. It is too early to assess whether this service will affect wait times. We did not collect patient identifiers and therefore could not track patients to verify whether patients had a face-to-face referral. We were unable to compare patient outcomes, including missed diagnoses, between eConsults and traditional referrals.

Another limitation includes our small dataset. Though to date the eConsult service has processed over 24,000 cases, including 787 rheumatology cases, the majority occurred after the end of the study period and were not included in this

Personal non-commercial use only. The Journal of Rheumatology Copyright $\odot$ 2018. All rights reserved. 
analysis. However, the usage data, including time to respond and referral avoidance, has remained very stable over time. As the eConsult service continues to broaden its geographic scope, increases patient volumes, and starts collecting patient identifiers, we will be able to address some of the above limitations and clearly identify the effect on patient outcomes.

The eConsultation service is a highly effective way to improve PCP access to rheumatology advice. Our service has not only reduced the need for face-to-face referrals but provides an opportunity to increase PCP capacity for managing this growing patient population.

\section{REFERENCES}

1. Hanly JG, Canadian Council of Academic Rheumatologists. Manpower in Canadian academic rheumatology units: current status and future trends. J Rheumatol 2001;28:1944-51.

2. Hanly JG. Physician resources and postgraduate training in Canadian academic rheumatology centers: a 5-year prospective study. J Rheumatol 2004;31:1200-5.

3. Deal CL, Hooker R, Harrington T, Birnbaum N, Hogan P, Bouchery E, et al. The United States rheumatology workforce: supply and demand, 2005-2025. Arthritis Rheum 2007;56:722-9.

4. Carriere G, Sanmartin C. Waiting time for medical specialist consultations in Canada, 2007. Health Rep 2010;21:7-14.

5. Shipton D, Badley EM, Bookman AA, Hawker GA. Barriers to providing adequate rheumatology care: implications from a survey of rheumatologists in Ontario, Canada. J Rheumatol 2002; 29:2420-5.
6. Wait Time Alliance. Time to close the gap: report card on wait times in Canada. [Internet. Accessed September 19, 2017.] Available from: www.waittimealliance.ca/wp-content/uploads/2014/06/ FINAL-EN-WTA-Report-Card.pdf

7. Widdifield J, Paterson JM, Bernatsky S, Tu K, Thorne JC, Ivers N, et al. Access to rheumatologists among patients with newly diagnosed rheumatoid arthritis in a Canadian universal public healthcare system. BMJ Open 2014;4:e003888.

8. Jong M, Kraishi M. A comparative study on the utility of telehealth in the provision of rheumatology services to rural and northern communities. Int J Circumpolar Health 2004;63:415-21.

9. Scheibe MM, Imboden JB, Schmajuk G, Margaretten M, Graf J, Chen AH, et al. Efficiency gains for rheumatology consultation using a novel electronic referral system in safety-net health setting. Arthritis Care Res 2015;67:1158-63.

10. Keely E, Liddy C, Afkham A. Utilization, benefits, and impact of an e-consultation service across diverse specialties and primary care providers. Telemed J E Health 2013;19:733-8.

11. Liddy C, Rowan MS, Afkham A, Maranger J, Keely, E. Building access to specialist care through e-consultation. Open Med 2013;7:e1-8.

12. Liddy C, Afkham A, Drosinis P, Joschko J, Keely E. Impact of and satisfaction with a new econsult service: a mixed methods study of primary care providers. J Am Board Fam Med 2015;28:394-403.

13. Ely JW, Osheroff JA, Gorman PN, Ebell MH, Chambliss ML, Pifer EA, et al. A taxonomy of generic clinical questions: classification study. BMJ 2000;321:429-32.

14. Harrington JT, Walsh MB. Pre-appointment management of new patient referrals in rheumatology: a key strategy for improving health care delivery. Arthritis Rheum 2001;45:295-300. 